\title{
Tsix et Xist, antisens et sens : du verlan dans l'inactivation du chromosome $X$ de la souris
}

Che» les mammifères, la compensation de dose, nécessaire compte tenu de l'existence d'un seul chromosome $\mathrm{X}$ chez le mâle, s'effectue grâce au processus d'inactivation du chromosome $\mathrm{X}[\mathrm{l}]\left(\mathrm{m} / \mathrm{s} 1996, n^{\circ} 3, p .409\right.$; $1997, n^{\circ} 5$, p. $723 ; 1998, n^{\circ} 10, p .1142$ et $\left.1999, n^{\circ} 11, p .1277\right)$, c'est-à-dire par l'extinction transcriptionnelle de la plupart des gènes d'un seul des deux chromosomes $\mathrm{X}$ de chaque cellule de l'embryon femelle. L'un ou l'autre des deux $X$ peut être choisi aléatoirement dans chaque cellule femelle, selon un mécanisme encore mystérieux. Le fait que ce processus ne soit pas initié dans les cellules de l'embryon mâle, qui ne comportent qu'un seul chromosome $X$, suggère l'existence d'un mécanisme de comptage qui perçoit le nombre de chromosomes X. Ces étapes de l'inactivation sont assurées par une région du chromosome $\mathrm{X}$ appelée centre d'inactivation (Xic), au sein de laquelle le gène Xist a été identifié. Ce gène exprime un long transcrit (15 kilobases chez la souris) qui reste nucléaire et ne code pas pour une protéine. Dans les cellules femelles adultes, l'ARN Xist est exprimé uniquement depuis le chromosome $\mathrm{X}$ inactif, il est particulièrement stable (demi-vie de 24 heures) et, par ARNFISH (hybridation in situ en fluorescence) reste associé au domaine nucléaire occupé par le chromosome $\mathrm{X}$ inactif. En revanche, dans les cellules embryonnaires avant la mise en place de l'inactivation, l'ARN de Xist est aussi exprimé depuis le chromosome $\mathrm{X}$ actif, mais il est instable et n'est détecté, par ARN-FISH, qu'associé au site primaire de transcription. Des expériences d'invalidation ont l'inactivation en cis joué par Xist, mais il est clair que ce dernier n'assure pas à lui seul toutes les fonctions du Xic. L'idée que la transcription antisens, récemment mise en évidence dans et autour du gène Xist [2-4], pourrait participer à certaines des fonctions du Xic a donc tout naturellement été évoquée. La région d'antitranscription s'étend sur 40 kilobases (kb) [2], ou plus (45kb) [4]. L'extrémité 3' d'un transcrit antisens a été identifiée $1,4 \mathrm{~kb}$ en amont de Xist par une analyse 3'RACE [3], mais l'anti-transcription reste détectable jusqu’à 5 kb en amont de Xist par ARN-FISH et par RT-PCR spécifique de brin [2], ce qui pourrait être expliqué par l'existence de plusieurs antitranscrits, puisque la visualisation, techniquement ardue, d'un transcrit de très grande taille n'a pas été réalisée. Au cours de la mise en place de l'inactivation, l'étude de l'expression de Xist et de son antitranscrit Tsix devrait permettre d'évaluer la possibilité d'une régulation croisée. L'absence de détection par RT-PCR de l'expression de Tsix dans les embryons murins de 5,5 jours post-coitum [3], stade légèrement postérieur à la mise en place de l'inactivation, s'accorderait bien avec un rôle répresseur ou déstabilisateur de Tsix sur Xist, dont la levée permettrait l'initiation de l'inactivation. Le modèle de différenciation des cellules ES femelles, qui permet d'étudier l'inactivation in vitro, a également été utilisé pour aborder cette question par ARNFISH. Il montre que la disparition du signal ARN-FISH de Tsix détecté par une sonde interne à Xist précède l'apparition des domaines recouverts d'ARN Xist qui signent l'initiation de l'inactivation [5]. Toujours dans le modèle des cellules ES différenciées, l'utilisation d'une sonde dans la région 5' de Tsix permet d'observer l'apparition de domaines recouverts d'ARN de Xist avant la disparition du signal de Tsix [4]. Les régions analysées dans ces deux études pourraient donc ne pas avoir la même signification fonctionnelle.

Une approche mutationnelle détaillée était donc nécessaire à l'élucidation du rôle de Tsix. Trois mutants relatifs à Tsix, obtenus dans des cellules ES, ont à ce jour été décrits (figure 1A): une délétion cre/lox $\mathrm{P}$ de $65 \mathrm{~kb}$ située en 3' de Xist [6], une délétion de 3,7 kb de la région initiatrice de Tsix [5], et une délétion de $3,0 \mathrm{~kb}$ portée par un YAC (yeast artificial chromosome) transgénique de $460 \mathrm{~kb}$ intégré sur un autosome [4]. Comme attendu, par ARN-FISH, aucune antitranscription n'est plus détectée depuis les chromosomes délétés pour le site d'initiation de Tsix $[4,5]$. Quant à l'expression de la transcription Xist sens, elle est totalement réprimée depuis le YAC transgénique porteur de la délétion de $3,0 \mathrm{~kb}$ [4] et partiellement réprimée depuis le chromosome $\mathrm{X}$ porteur de la délétion cre/loxp [6], alors que cet effet n'a pas été rapporté pour la délétion de $3,7 \mathrm{~kb}$ [5]. La différence des phénotypes des délétions de 3,0 et de $3,7 \mathrm{~kb}$ suggère que l'effet de séquences d'ADN régulatrices pourrait se superposer à l'action de Tsix sur la régulation de Xist. Cependant, après différenciation des cellules ES porteuses d'un Xic muté et d'un Xic non muté, les trois chromosomes $X$ mutants conservent tous la capacité d'amorcer le processus d'inactivation et de conduire à la formation d'un 


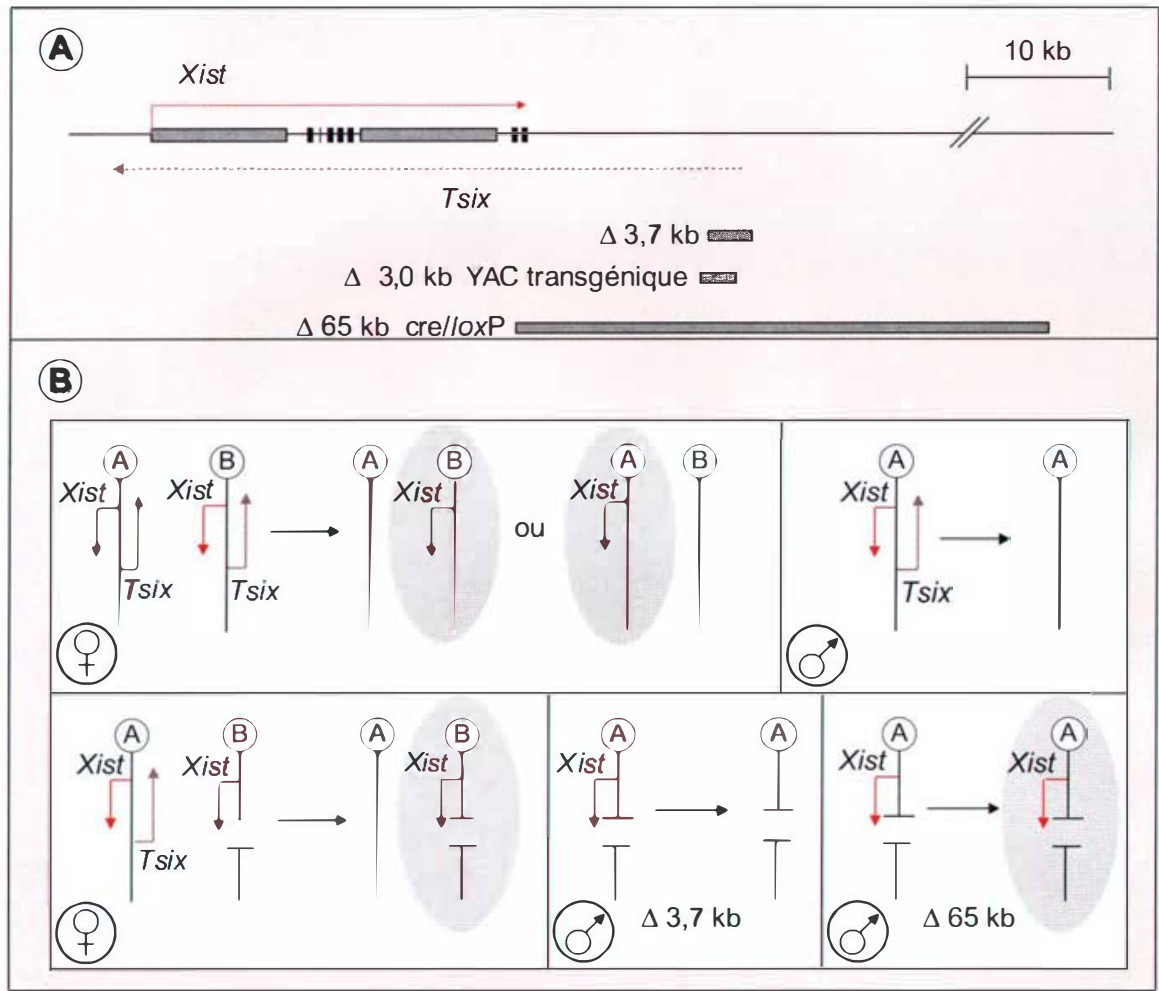

Figure 1. A. Carte des régions géniques Xist sens et Tsix antisens. La région $d^{\prime}$ antitranscription est figurée en pointillés en raison d'incertitudes concernant ses frontières et sa continuité (voir texte). L'étendue des trois mutations publiées concernant Tsix figure sous la carte [1-3]. B. Effet des délétions de Tsix sur l'inactivation du chromosome $X$. En haut à gauche, illustration de l'inactivation aléatoire dans les cellules femelles sauvages. L'un ou l'autre des chromosomes $X$ peut être choisi pour être inactivé. II sera alors recouvert par l'ARN de Xist (figuré par le halo gris) tandis que Tsix s'éteint sur les deux allèles et que le gène Xist s'éteint sur le chromosome actif. En haut à droite, absence d'inactivation dans les cellules mâles sauvages. En bas à gauche, dans les cellules femelles, les mutants pourTsix inactivent systématiquement le chromosome $X$ porteur de la mutation, jamais plus le chromosome $X$ nonmuté. En bas à droite: dans une cellule mâle, le chromosome $X \Delta 3,7 \mathrm{~kb}$ se comporte comme un chromosome $X$ non muté, alors que le chromosome $X$ $\Delta 65 \mathrm{~kb}$ est capable de s'inactiver même s'il est seul dans la cellule.

domaine recouvert de transcrit Xist stable. Les mécanismes régulateurs du gène Xist avant et après la mise en place de l'inactivation diffèrent donc fondamentalement. Mais l'observation la plus surprenante est que l'induction de l'inactivation du chromosome $\mathrm{X}$ porteur du Xic sauvage n'est plus jamais observée (figure IB). La conclusion minimale est que Tsix, ou les régions génomiques délétées, participent directement ou indirectement au choix du chromosome X à inactiver. Mais qu'en est-il du proces- sus de comptage qui empêche l'inactivation lorsqu'une cellule n'a qu'un seul chromosome $X$ ? Dans ce contexte "solitaire", les délétions de $3,7 \mathrm{~kb}$ [5] et de $65 \mathrm{~kb}$ [6] ont des effets différents. Le chromosome $\mathrm{X}$ porteur de la délétion de $3,7 \mathrm{~kb}$ reste actif après différenciation des cellules ES [5] : le comptage reste donc opérant sur ce chromosome. Toutefois, le chromosome $\mathrm{X}$ porteur de la délétion de $65 \mathrm{~kb}$ n'est plus sensible au comptage: il peut déclencher un processus d'inactivation apparem- ment normal même en l'absence d'un autre Xic dans la cellule [6]. L'interprétation la plus simple est que Tsix n'est pas directement impliqué dans le processus de comptage qui nécessiterait des éléments plus distaux.

La question du choix lors de la compensation de dose chez les mammifères pourrait donc trouver une solution dans l'étude de la transcription antisens au locus Xist. Comme pour certains gènes soumis à empreinte (pour revue, voir [7]), l'antitranscription s'affiche en régulateur de l'expression génique chez les mammifères, et pourrait agir par régulation de la structure chromatinienne, par compétition entre promoteurs, et par interaction moléculaire directe des transcrits sens et antisens.

1. Heard E, Clerc P, Avner P. X-chromosome inactivation in mammals. Annu Rev Genet 1997; $31: 571-610$.

2. Lee JT, Davidow IS, Warshawsky D). Tsix, a gene antisense to Xist at the X-inactivation centre. Nat Genet 1999; $21: 400-4$.

3. Mise N, Goto Y, Nakajima N, Takagi N. Molecular cloning of antisense tuanscripts of the mouse Xist gene. Biochem Biophys Res Commun $1999 ; 258: 537-41$.

4. Debrand E, (hureau C, Amaud I), Avner P, Heard E. Functional analysis of the 1)XPas 34 locus, a 3' regulator of Xist expression. Mol Cell Biol 1999; 19: 8513-25.

5. Lee JT, Lu N. Targeted mutagenesis of Tsix leads to nonrandom X inactivation. Ciell 1999; 99 : 47-57.

6. Clerc P. Avner P. Role of the region 3' to Xist exon 6 in the counting process of X-chromosome inactivation. Nat Genet 1998; 19: 249-53.

7. Tilghman SM. The sins of fathers and mothers: genomic imprinting in mammalian development. Cell 1999; 96: 185-93

\section{Philippe Clerc}

Unité de génétique moléculaire murine, Institut Pasteur, 25, rue du DocteurRoux, 75015 Paris, France. 